\title{
Dispositionalism and the Modal Operators
}

DAVID YATES

University of Oxford

\section{Introduction}

Actualists of a certain stripe hold that metaphysical possibility and necessity are grounded in the powers of actual things. I shall refer to this theory as dispositionalism for brevity. ${ }^{1}$ At a first pass, < possibly, David Yates is a dancer $>$, if true, is made true by powers certain actual things have, or had, such that the manifestations of those powers would have resulted in or constituted my being a dancer. It need not be the case that I alone have the powers in question. Perhaps some of the relevant powers belong to dance teachers I never had; or to music I never heard; perhaps they are powers to bring about mutations in my DNA prior to birth, such that had those powers manifested, I would have been less clumsy and more inclined to dance than I actually am.

Given dispositionalism, the truthmakers of true modal propositions are the powers of actual things. Dispositionalists are, to borrow Contessa's phrase, hardcore actualist, in that they seek to explain modality without possible worlds - going directly, so to speak, from the powerful to the possible. $^{2}$ The resulting theory is non-reductive, in that the explanans are themselves, in a sense, irreducibly modal. We might say that powers are essentially such as to manifest, producing tokens of their manifestation types, under characteristic stimulus conditions. Or-if this is different-we could say that powers are, of their nature, primitive truthmakers of a range of counterfactuals relating them to their stimuli and manifestations. It is now customary to deny that powers have non-powers as stimulus

1 I focus on de dicto modality, rather than the more controversial de re variety, for reasons of exposition. My usage of 'dispositionalism' follows Contessa (2010). I use angle brackets to refer to propositions, so that $\langle p\rangle$ refers to the proposition that $p$.

2 Contessa (2010). 
conditions, and say instead that they manifest when combined with reciprocal partner powers. ${ }^{3}$ Powers so conceived are, of their natures, such as to produce a range of different manifestations when combined with each other in various ways.

Extant discussions of dispositionalism focus on its material adequacy: are there enough powers to account for all the metaphysical possibilities we wish to countenance? Those who think not point to certain intuitive unactualized possibilities and argue that no actual thing has the power to bring them about. Cameron, for instance, argues that it is possible that none of the actual contingent existents exists, but there is no way to account for this possibility in terms of the powers of any subset of those very things. ${ }^{4}$ Pruss attempts to solve this problem by positing a necessarily existent God as bearer of the powers that ground modality. ${ }^{5}$ As Cameron points out, however, this move renders modality epistemically intractable. ${ }^{6}$ Why should knowing God's powers be any easier than knowing what is and is not possible? A better option for dispositionalists is to say that modality, qua grounded in powers, is not accessible to a priori reflection, and bite the bullet: no power, no possibility. If you want to know whether something is possible, ask a scientist. ${ }^{7}$

My focus in this paper will be on the formal adequacy of dispositionalism. I draw attention to certain actualized possibilities for which no corresponding powers exist, and argue from there that dispositionalism, as explicitly defined by several of its chief proponents, is formally inadequate. I offer a modification to dispositionalism which secures formal adequacy and through this suggest a principled restriction on the role of powers in grounding modality. My aim is not to refute dispositionalism, but to amend it, and I see no reason why proponents of the dispositionalist theory detailed below should not embrace the amendments I suggest.

\section{A Simple Dispositional Interpretation of the Modal Operators}

To get from a truthmaking theory for modal truths to a modal logic, the theory must supply a way of defining the modal operators. Dispositionalists typically have simple existential definitions in mind, as the following proposals show:

\footnotetext{
Martin (2008).

Cameron (2008).

Pruss (2002, 2011).

See his (2008), pp. 275-276.

For a well-developed version of this strategy, see Contessa (2010).
} 
State of affairs $S$ is possible iff there is some actual disposition $d$ whose manifestation is (or includes) S. (Williams and Borghini (2008), p. 26) ${ }^{8}$

[S]ome proposition or truth $\mathrm{T}$ is possible just in case there is some actually instantiated property (or property complex) that is a power for some other property (or property complex) that would be a truthmaker for T. (Jacobs (2010), p. 236)

It is possible that $p$ if and only if something has (or some things have) an iterated potentiality for it to be the case that $p$. (Vetter, forthcoming)

Despite certain differences of detail which we will gloss over here, all the above authors appeal to powers to define the possibility operator, and the common thread is the proposal that $\nabla p$ iff something has, or some things have, the power to bring it about that $p$. Williams and Borghini go on to restrict the existential claim in the definiens to instantiated powers; Jacobs takes powers to be immanent universals; and Vetter is also explicit that the relevant powers are powers of actual things. There need not be any single actually instantiated power whose manifestation under some circumstances would have been that $p$, in order for it to be the case that $\diamond p .^{9}$ A plurality of things collectively have the power to bring it about that $p$ iff there are ways of combining them such that had they been so combined, they would have produced a truthmaker for $\langle p\rangle$. Given the interdefinability of the operators, we can define the necessity operator in terms of a negative existential: $\square p$ iff nothing has, and no things have, the power to bring it about that $\neg p$.

For clarity in what follows, let us express these definitions a little more formally. Rather than quantify over things, I shall use second-order quantification over powers. Let ' $\exists \phi>[p](\phi)$ ' abbreviate <there is a $\phi$ such that $\phi$ is a power to bring it about that $p>$, taking ' $>[p]$ ' to denote the predicate ' power to bring it about that $p$ '. Those who prefer to think of dispositionalism in terms of empowered particulars may read ' $\exists \phi>[p](\phi)$ ' as shorthand for $\langle\exists x \exists \phi\{\phi(x) \wedge>[p](\phi)\}>$. For simplicity I avoid plural quantification over powers, but the reader should bear in mind the remarks of the preceding paragraphs when interpreting these formalizations - there need be no single power whose manifestation would be a truthmaker for $\langle p\rangle$. We now have the following definitions:

8 Williams and Borghini modify this proposal to accommodate higher-order dispositions, but the details need not concern us here, as the modified account does not escape the criticisms of the present work.

9 Thus Jacobs (2008) allows for a "chain of such connections" between powers and truthmakers for the propositions whose possibility they ground; and Vetter (forthcoming) refers to "iterated potentialities", which are potentialities to produce something that has the potentiality... to produce a truthmaker for $\langle p\rangle$. 


$$
\begin{aligned}
\mathrm{POSS}_{1}: \diamond p & \equiv \exists \phi>[p](\phi) \\
\mathrm{NEC}_{1}: \square p & \equiv \neg \exists \phi>[\neg p](\phi)
\end{aligned}
$$

Note that $\left(\mathrm{NEC}_{1}\right)$ is a substitution instance of $\left(\mathrm{POSS}_{1}\right)$, given that $\square p \equiv \neg \diamond \neg p$. Given ( $\left.\mathrm{POSS}_{1}\right), \diamond \neg p \equiv \exists \phi>[\neg p](\phi)$, which we negate to get: $\neg \diamond \neg p \equiv \neg \exists \phi>[\neg p](\phi)$. According to this theory, the existence of a power to bring it about that $p$ is both necessary and sufficient for $<p>$ 's possible truth; let us therefore refer to it as strong dispositionalism.

\section{The Formal Inadequacy of Strong Dispositionalism}

In this section I argue that the strong dispositionalist interpretations of the modal operators results in a formally inadequate modal logic. Before proceeding, let us consider some familiar conditions on formal adequacy. In the now standard way, we can obtain modal logics with different formal properties by adding various axioms to standard propositional logic:

- $\mathrm{K}: \square(p \supset q) \supset(\square p \supset \square q)$

- $\mathrm{T}: \square p \supset p$

- 4: $\square p \supset \square \square p$

- 5: $\diamond p \supset \square \diamond p$

To get system $\mathrm{K}$, we add axiom (K) to propositional logic. ${ }^{10}$ System $\mathrm{T}$ results from the addition of (T) to $\mathrm{K} ; \mathrm{S} 4$ and $\mathrm{S} 5$ result, respectively, from adding (4) and (5) to T. Let us first think of these systems in terms of Kripke semantics, which provides a useful contrast with dispositionalism. A Kripke model is a triple consisting of a set of possible worlds, a relation $R$ (accessibility) between worlds, and a satisfaction relation between worlds and formulae (intuitively, truth-at-a-world). In Kripke semantics, $<\square p>$ is true at a world $w$ iff $\langle p>$ is true at every world accessible from $w ;\langle\diamond p>$ is true at $w$ iff $\langle p\rangle$ is true at some world accessible from $w$.

The above axioms may be distinguished in terms of the constraints they place on $R$. (K) places no constraints on $R$. (T) is equivalent to $\langle p \supset \diamond p\rangle$, whose general truth requires every world to be accessible from itself, so (T) holds iff $R$ is reflexive. Similarly, (4) holds iff $R$ is transitive, i.e. if $R\left(w_{1}\right.$, $\left.w_{2}\right)$ and $R\left(w_{2}, w_{3}\right)$ then $R\left(w_{1}, w_{3}\right)$; and (5) holds iff $R$ is Euclidean, i.e. if $R$ $\left(w_{1}, w_{2}\right)$ and $R\left(w_{1}, w_{3}\right)$ then $R\left(w_{2}, w_{3}\right)$. In the system $\mathrm{S} 5, R$ is an equivalence relation: every world is accessible from every world, so that possibility and

10 And a rule of inference, Necessitation: $|-p \supset|-\square p$, which I have omitted for brevity. 
necessity, respectively, are equivalent to truth at some, and every, possible world simpliciter. For strong dispositionalists, the axioms place corresponding constraints on the availability of powers: $(\mathrm{K})$ holds iff, if there is no power to bring about that $\neg(p \supset q)$ and no power to bring it about that $\neg p$, then there is no power to bring it about that $\neg q$. (T) holds iff, if $\langle p\rangle$ is true then there is a power to bring it about that $p$. (4) holds iff, if there is no power $\phi$ to bring it about that $\neg p$, then there is no power $\varphi$ to bring it about that there is such a $\phi$. Finally, (5) holds iff, if there is a power $\phi$ to bring it about that $p$, then there is no power $\varphi$ to bring it about that there is no such $\phi$.

It is widely accepted that at least system $\mathrm{T}$ is required for a logic of metaphysical modality. It is intuitively clear, for instance, that unless (T) holds, ' $\square p$ ' does not express <it is metaphysically necessary that $p>$. It is also standard to hold that for metaphysical modality, $\mathrm{T}$ is not strong enough, and must be supplemented by either (4) or (5). I shall not be too concerned with the details here, for my arguments against the formal adequacy of strong dispositionalism do not presuppose any controversial conditions on formal adequacy. Strong dispositionalism validates neither $(\mathrm{K})$ nor (T), rendering it formally inadequate. For reasons of exposition, I first argue that strong dispositionalism does not validate $(\mathrm{T})$, then for completeness show that it does not validate $(\mathrm{K})$ either.

Consider $<2+2=4>$. The world contains things with the power to bend, break, attract and repel, but nothing with the power to bring it about that $\neg(2+2=4)$. Not even God can do that. As required, then, we have $\neg \exists \phi>[\neg(2+2=4)](\phi)$, hence from $\left(\mathrm{NEC}_{1}\right), \square(2+2=4)$ as required. Unfortunately, it is also true that there is nothing with the power to bring it about that $2+2=4$. This is not the sort of thing powers are powers to do. No network of powers has a truthmaker for $<2+2=4>$ as its manifestation. There are powers such that were they to manifest, it would be the case that $2+2=4$ - all powers are like that. This is not the same, however, as there being a power to bring it about that $2+2=4$. We therefore have: $\neg \exists \phi>[(2+2=4)](\phi)$, from which, given $\left(\mathrm{POSS}_{1}\right)$, we can deduce that $\neg \diamond(2+2=4)$. According to strong dispositionalism, it is both necessary and not possible that $2+2=4$. Given the interdefinability of the operators, $\angle \square(2+2=4)>$ entails $<\neg \diamond \neg(2+2=4)>$; and similarly $\langle\neg \diamond(2+2=4)>$ entails $\langle\square \neg(2+2=4)>$. Given strong dispositionalism, for any arithmetic proposition $p$ we have: $\square p$, hence $\neg \diamond \neg p$; but also $\neg \diamond p$, hence $\square \neg p$.

These results show that strong dispositionalism is formally inadequate. Both $(\mathrm{T})$ and $(\mathrm{K})$ are invalid for the same reason: given strong dispositionalism, some necessary propositions are not true, or equivalently, some true propositions are not possible. If we write (T) as: $\square p \supset p$, then $<\neg(2+2=4)>$ is a counterexample, for according to strong dispositionalism, $\square \neg(2+2=4)$, yet of course $\neg \neg(2+2=4)$. If you prefer, write (T) in its contrapositive form: $p \supset \diamond$ p. 
Then $<2+2=4>$ is a counterexample, for $2+2=4$, yet given strong dispositionalism, $\neg \diamond(2+2=4)$. To see that axiom $(\mathrm{K}): \square(p \supset q) \supset(\square p \supset \square q)$ is not valid, let $p$ be $<\neg(2+2=4)>$, and let $q$ be $<$ grass is green $>$. On this substitution, $\neg \exists \phi>[\neg\{p \supset q\}](\phi)$, because $\neg \exists \phi>[p \wedge \neg q](\phi) .{ }^{11}$ Hence $\square(p \supset q)$. However, it is not the case that $\square p \supset \square q$. On strong dispositionalism, $\square p$, because there is no power to bring it about that $2+2=4$. Since bringing it about that $p$ is equivalent to bringing it about that $\neg \neg p, \quad\langle\neg \exists \phi\rangle[2+2=4](\phi)\rangle$ entails $\langle\neg \exists \phi\rangle[\neg \neg(2+2=4)](\phi)>$, from which it follows that $\square p$. However, $\neg \square q$, because plausibly $\exists \phi>[\neg$ (grass is green $)](\phi)$.

\section{Objections and Replies}

(A) A plenitude of powers. Suppose that for any $x$ and any property $\mathrm{P}$ such that $\mathrm{P}(x), x$ has the power to instantiate $\mathrm{P}$. Clearly, everything has the property of being an $x$ such that $2+2=4$; then it is possible that $2+2=4$ because every $x$ has the power to be an $x$ such that $2+2=4$. The principle of plenitude guarantees a power for every actualized possibility. Now the power to be such that $2+2=4$, whatever it might be, is not the power to bring it about that $2+2=4$. A plurality of powers explains $\langle p>$ 's possibility by being such that their mutual manifestation would include a truthmaker for $\langle p>$. The powers that metaphysically explain $\langle p>$ 's possible truth would causally explain $<p>$ 's truth if combined in the appropriate way. The putative universally instantiated power to be an $x$ such that $2+2=4$, by contrast, has no connection with the truthmaker for $<2+2=4>$, whatever that might be. It has neither reciprocal partner powers, nor any other condition on its manifestation, for it is never unmanifested.

To say the possible truth of $<$ David Yates is a dancer $>$ is grounded in powers to bring it about that I am a dancer involves a substantive empirical claim about the causal structure of the world. By contrast, the claim that everything has the always activated, irreducibly modal power to be such that $2+2=4$ is hardly different from the claim that it is possible that $2+2=4$. What makes the non-reductive explanation of modality offered by dispositionalism informative is that we have independent epistemic access to the explanantia. Positing ad hoc powers of a different kind, which are not amenable to scientific investigation, and whose sole function is to mop up a residue of possibilities left unexplained by those that are, is unhelpful. The principle of plenitude promises new explanantia for the residual possibilities, but delivers little more than a restatement of the explananda using a different term.

11 Any power to bring it about that $\neg\{\neg(2+2=4) \supset$ (grass is green) $\}$ would be a power to bring it about that $\neg(2+2=4)$, for otherwise the conditional is true; there is no such power. 
(B) The counterfactual route. Having first endorsed the strong dispositionalist theory detailed in $\S 2$, Jacobs then appeals to the following definitional equivalences: ${ }^{12}$

$$
\begin{gathered}
\mathrm{POSS}_{2}: \diamond p \equiv \neg(\tau \Rightarrow \neg p) \\
\mathrm{NEC}_{2}: \quad \square p \equiv \tau \Rightarrow p
\end{gathered}
$$

Here ' $~ \tau$ ' denotes an arbitrary tautology, and ' $\Rightarrow$ ' the counterfactual conditional. Intuitively, we can treat ' $\tau$ ' as a placeholder for any proposition we like, so that according to the above definitions, $\square p$ iff whatever else were the case, it would be the case that $p$; and $\diamond p$ iff it is false that whatever else were the case, it would not be the case that $p .^{13}$ Accepting the extensional adequacy of $\left(\mathrm{POSS}_{2}\right)$ and $\left(\mathrm{NEC}_{2}\right)$ does not of course commit one to any form of dispositionalism. Jacobs' idea is to hold that counterfactuals have dispositional truthmakers, and then appeal to $\left(\mathrm{POSS}_{2}\right)$ and $\left(\mathrm{NEC}_{2}\right)$ to get from counterfactuals to modality. Roughly, $\langle p \Rightarrow q\rangle$ is true iff $\langle p\rangle$ represents a way of combining certain reciprocal partner powers and $\langle q\rangle$ represents a mutual manifestation of those powers, so combined. If counterfactuals in general have dispositional truthmakers, then given that the operators can be counterfactually defined, modal truths will likewise have dispositional truthmakers.

The possible and necessary truth of $<2+2=4>$ are analysed by (i) $<\neg[\tau \Rightarrow \neg(2+2=4)]>$ and (ii) $<\tau \Rightarrow(2+2=4)>$ respectively. Given the extensional adequacy of $\left(\mathrm{POSS}_{2}\right)$ and $\left(\mathrm{NEC}_{2}\right)$, we may safely assume that (i) and (ii) are true; what is of interest is whether they have dispositional truthmakers, as it is crucial for Jacobs that they do. Given what he has to say about the relationship between powers and counterfactuals, however, it is difficult to see how they could. Suppose we treat ' $\tau$ ' as a placeholder for propositions that represent any combination of powers we like. Then given Jacobs' truth-conditions for counterfactuals, (i) is true iff it is false that every combination of powers would bring it about that $\neg(2+2=4)$, and (ii) is true iff every such combination would bring it about that $2+2=4$. This counterfactual version of dispositionalism is not, as Jacobs seems to suppose, equivalent to strong dispositionalism. On the current proposal, $\langle p\rangle$ is possible iff not every power is a power to bring it about that $\neg p$, and $\langle p\rangle$ is necessary iff every power is a power to bring it about that $p$. Undoubtedly, not every power is a power to bring it about that $\neg(2+2=4)$, so (i) comes out true. Since there is no power to bring it about that $2+2=4$, however, a fortiori it is not the case that every

Jacobs (2010), p. 18.

13 Williamson (2005) offers a rather more perspicuous explicitly quantificational analysis: $\square A \equiv \forall p(p \Rightarrow A)$. 
power is a power to bring it about that $2+2=4$, and (ii) comes out false. Given that (ii) is true, it cannot have the kind of dispositional truthmaker that Jacobs requires.

(C) Straw man. The reader may suspect that I attack a straw man. Contessa, when offering a rough characterisation of dispositionalism, gives not a biconditional but a conditional form, according to which powers are sufficient, but not necessary, for possibility: $\exists \phi>[p](\phi) \supset \diamond p .{ }^{14}$ This sufficiency claim is not vulnerable to the objections I have raised. Contessa aims, however, to defend dispositionalism from Cameron's aforementioned objection that there is no power available to ground the possibility that none of the actual concrete existents exists. The sufficiency conditional above is not vulnerable to Cameron's objection either, which is a putative counterexample only to the converse necessity conditional. Tellingly, having characterised dispositionalism as a sufficiency claim, Contessa goes on to ask: "[b]ut can dispositionalists account for all modal truths in this way? Cameron suggests they can't." 15 Hence, what is at stake for both Contessa and Cameron is a biconditional form of dispositionalism such as explicitly endorsed by the authors quoted in $\S 2: \diamond p \equiv \exists \phi>[p](\phi)$.

That strong dispositionalists endorse the preceding biconditional does not suffice to save me from the straw man objection, for it might be said that they implicitly restrict the scope of the propositional quantifier, thereby restricting the domain of propositions whose modal status powers are claimed to ground. At a first pass, we might say that powers account for the modal status of empirical propositions only; for those that concern abstracta, we need a different truthmaking theory. We might then develop a formally adequate domain-specific modal logic by similarly restricting quantification in its axioms. ${ }^{16}$ Unfortunately, there are true empirical propositions whose truth there is no power to bring about. Here is one: the total mass-energy $\mathrm{E}_{\mathrm{T}}$ of the universe is $\sum$. Assume $\mathrm{E}_{\mathrm{T}}$ is conserved, come what may: $\neg \exists \phi>\left[\neg\left(\mathrm{E}_{\mathrm{T}}=\sum\right)\right](\phi)$. It follows from this, by $\left(\mathrm{NEC}_{1}\right)$, that $\square\left(\mathrm{E}_{\mathrm{T}}=\sum\right)$. It is also the case, however, that $\neg \exists \phi>\left[\left(\mathrm{E}_{\mathrm{T}}=\sum\right)\right](\phi)$, because no actual thing has the power to bring it about that $\mathrm{E}_{\mathrm{T}}=\sum$ either. It follows from $\left(\mathrm{POSS}_{1}\right)$ that $\neg \diamond\left(\mathrm{E}_{\mathrm{T}}=\sum\right) .<\mathrm{E}_{\mathrm{T}}=\sum>$ is both necessary and not possible. Again, from the interdefinability of the operators, we may conclude the same concerning its negation. There are other cases. Write down a conjunction of positive existential propositions, one for each actual power. Unless the powers whose existence we correctly assert are powers to bring about their own existence,

Contessa (2010), p. 342.

16 I do not claim that the suggested demarcation into empirical and non-empirical is straightforward, or that a modal logic restricted to empirical propositions would be worth formulating.
} 
the possible truth of this conjunction cannot be grounded in a power to bring about its truth.

If the propositional quantifier is to be restricted, then we shall have to think of an alternative way of restricting it. In $\$ 5$, I propose an amendment to strong dispositionalism that secures formal adequacy. The amendment entails that the existence of a power to bring it about that $p$ is sufficient, but not necessary, for the possible truth of $\langle p\rangle$, but also entails that there is a restricted domain of propositions for the possible truth of which the corresponding powers are both necessary and sufficient. The restricted scope biconditional, however, while plausibly true, does not define the operators of a domain-specific modal logic.

\section{Solving the Formal Adequacy Problem}

The problem for strong dispositionalism is that $\langle p\rangle$ does not entail $\langle\vee p\rangle$, or equivalently, that $\langle\square p\rangle$ does not entail $\langle p\rangle$. The solution I shall consider is to build $\langle p>$ 's truth into the definitions of the operators, so that by stipulation the relevant entailments hold. We can do so by disjoining the definiens of $\left(\mathrm{POSS}_{1}\right)$, and conjoining that of $\left(\mathrm{NEC}_{1}\right)$, with $<p>$. ${ }^{*}$ Let us refer to the following theory as weak dispositionalism:

$$
\begin{aligned}
\mathrm{POSS}_{3}: \diamond p & \equiv\{p \vee \exists \phi>[p](\phi)\} \\
\mathrm{NEC}_{3}: & \square p \equiv\{p \wedge \neg \exists \phi>[\neg p](\phi)\}
\end{aligned}
$$

According to $\left(\mathrm{POSS}_{3}\right),<p>$ is possible iff $<p>$ is true or there is a power to bring it about that $p$. Given interdefinability, we get $\left(\mathrm{NEC}_{3}\right):<p>$ is necessary iff $\langle p\rangle$ is true and there is no power to bring it about that $\neg p .{ }^{17}$ I shall discuss some philosophical implications of these definitions in $\S 6$, but for now let us examine some of their formal consequences.

Let us begin, as before, with axiom (T): $p \supset>p$. I need say nothing about this other than that it follows logically from $\left(\mathrm{POSS}_{3}\right)$ that $p \supset \vee p$. What about $(\mathrm{K})$ ? Suppose that $\square(p \supset q)$. From $\left(\mathrm{NEC}_{3}\right)$, it follows that $(p \supset q) \wedge \neg \exists \phi>[\neg(p \supset q)](\phi)$. Now suppose in addition that $\square p$; hence $p \wedge \neg \exists \phi>[\neg p](\phi)$. Given $\left(\mathrm{NEC}_{3}\right)$, the following are logical consequences of $\langle\square(p \supset q) \wedge \square p>$ : (i) $\langle p>$; (ii) $\langle p \supset q>$;

[Correction added on 20 April 2015, after first online publication: The author added the following sentence: Pruss $(2002,2011)$ also suggests a disjunctive approach to possibility, but offers little by way of explicit argument for it. I arrived at this theory independently, as a solution to the formal adequacy problem discussed above. However, see Pruss (2011), pp. 165-171, for remarks on the possibility of the actual similar to some of those I make in $\S 6$.] 
(iii) $\langle q\rangle$; (iv) $\langle\neg \exists \phi\rangle[\neg p](\phi)>$; (v) $<\neg \exists \phi\rangle[\neg(p \supset q)](\phi)>$. In English: given weak dispositionalism, $\langle\square(p \supset q) \wedge \square p>$ entails (i) that $\langle p\rangle$ and $\langle p \supset q\rangle$ are true, hence that $\langle q\rangle$ is true, and (ii) that there is no power to bring it about that either $\langle p\rangle$ or $\langle p \supset q\rangle$ is false. Now suppose for reductio that there is a power to bring it about that $\neg q$. Were this power activated, it would thereby bring it about that either $\langle p\rangle$ or $\langle p \supset q\rangle$ were false, since $\langle p\rangle$ and $\langle p \supset q\rangle$ are true and entail $\left\langle q>\right.$. ${ }^{18}$ By hypothesis, there is no power to bring about the falsity of either $\langle p\rangle$ or $\langle p \supset q\rangle$. Hence $\neg \exists \phi\rangle[\neg q](\phi)$. Since $\langle q\rangle$ is true, we may conclude that $q \wedge \neg \exists \phi\rangle[\neg q](\phi)$, from which $\langle\square q\rangle$ follows by $\left(\mathrm{NEC}_{3}\right)$. Hence $[\square(p \supset q) \wedge \square p] \supset \square q$, or equivalently, $\square(p \supset q) \supset(\square p \supset \square q)$. On weak dispositionalism, both $(\mathrm{T})$ and $(\mathrm{K})$ are valid.

It is relatively easy to show that (4) is valid on weak dispositionalism, and instructive to see why. ${ }^{19}$ Recall (4): $\square p \supset \square \square p$. Applying $\left(\mathrm{NEC}_{3}\right)$, we can write $\langle\square \square p>$ as follows: $\square p \wedge \neg \exists \varphi\rangle[\neg \square p](\phi)$. Substituting into (4), we get: $\square p \supset\{\square p \wedge \neg \exists \varphi>[\neg \square p](\varphi)\}$. Now it is trivial that $\square p \supset \square p$, so the validity of (4) turns on whether $\square p \supset \neg \exists \varphi>[\neg \square p](\varphi)$. Iterating $\left(\mathrm{NEC}_{3}\right)$ to substitute for ' $\square p$ ', our question then becomes: does (A) entail (B)?

$$
\begin{aligned}
& \text { (A) } p \wedge \neg \exists \phi>[\neg p](\phi) \\
& \text { (B) } \neg \exists \varphi>\neg[\{p \wedge \neg \exists \phi>[\neg p](\phi)\}](\varphi)
\end{aligned}
$$

In English: does $<p$, and there is no power $\phi$ to bring it about that $\neg p>$ entail that there is no power $\varphi$ to bring it about that $<p$, and there is no power $\phi$ to bring it about that $\neg p>$ is false? Suppose there is such a power $\varphi$. In order to bring it about that $\neg\{p \wedge \neg \exists \phi>[\neg p](\phi)\}, \varphi$ must either bring it about that $\neg p$, or bring it about that $\exists \phi>[\neg p](\phi)$. It is useful to reflect at this point on what all this actually means: in order to bring it about that $\langle p\rangle$ is not necessary, $\varphi$ must either bring it about that $\langle p\rangle$ is false, or bring it about that there is a power to bring it about that $\langle p\rangle$ is false. It follows from (A) that $\neg \exists \phi>[\neg p](\phi)$, so we can rule the first option out. Given the way we are understanding <there is a power to bring it about that $p>$, however, $\langle\neg \exists \phi\rangle[\neg p](\phi)>$ entails $\langle\neg \exists \varphi\rangle[\exists \phi>[\neg p](\phi)](\varphi)>$, so we can rule out the second option too, and (4) is valid on weak dispositionalism.

Think of it as follows. The powers of actual things determine a branching structure - all the ways the world would have been, had those powers

\footnotetext{
18 On the assumption, which I take to be uncontroversial, that there is no power to bring about a contradiction. for brevity. The proof for (5) is less compelling than that for (4), because it depends on some contentious principles.
} 
been combined in various ways. ${ }^{20}$ Suppose that $\langle p\rangle$ is true, and that nothing throughout the history of our world ever has the power $\phi$ to bring it about that $\neg p$. Could anything have the unmanifested power $\varphi$ to bring it about there is a power $\phi$ to bring it about that $\neg p$ ? No. If there is such a $\varphi$, then there is a branch leading from actuality to a state of affairs in which $\neg p$. This, however, is all it means for there to be a power to bring it about that $\neg p$. Such transitive cases of bringing about, where a power brings about the truth of a proposition by first bringing about some further configuration of powers, are built into the way we are thinking about how powers bring about the truth of propositions. We can depict the situation as follows:

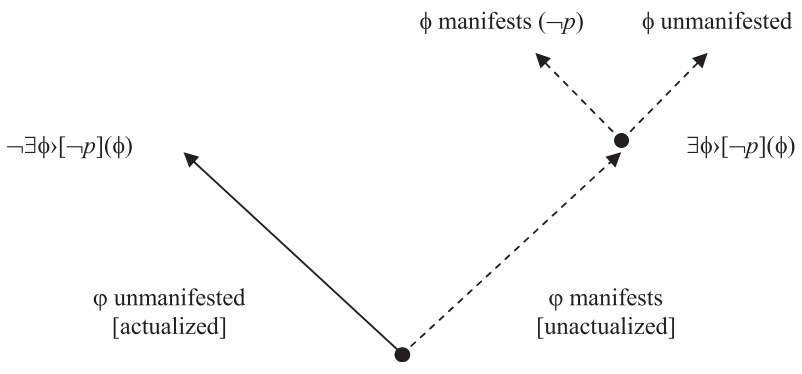

A moment's reflection will show that the above diagram is incoherent. For it to be the case that $\neg \exists \phi>[\neg p](\phi)$ in the actualized branch, it must be the case that at no point in the history of the world is there a power to bring it about that $\neg p$. However, the world and the unactualized right hand branch in which (putatively) $\exists \phi>[\neg p](\phi)$ have a common origin - the point at which the hypothetical power $\varphi$ fails to manifest. It follows that there is a path leading from that point in our history to a branch in which $\neg p$ (the ' $\phi$ manifests' branch). That, however, is all it is for there to be a power to bring it about that $\neg p$, which is contrary to our initial assumption that no such power exists. ${ }^{21}$

In Kripke models, (4) holds only if the accessibility relation $R$ is transitive. We have a corresponding result in the present case, for (4) holds iff $\neg \exists \phi>[\neg p]$ $(\phi) \supset \neg \exists \varphi>[\exists \phi>[\neg p](\phi)](\varphi)$, or equivalently: $\exists \varphi>[\exists \phi>[\neg p](\phi)](\varphi) \supset \exists \phi>[\neg p](\phi)$. Because nothing turns on negation in the preceding formula, the general condition (4) places on the domain of powers is that $\exists \varphi>[\exists \phi>[p](\phi)]$ $(\varphi) \supset \exists \phi>[p](\phi)$. In English: if there is a power $\varphi$ to bring it about that there is a power $\phi$ to bring it about that $p$, then there is a power $\phi$ to bring it about that $p$. As we have seen, this sort of transitivity is built into our pres-

Williams and Borghini (2009) make much use of the branching image.

21 There is a further reason, given Aristotelianism about universals, to doubt that there could be a power $\varphi$ to bring about an instance of an uninstantiated power $\phi$. Suppose powers are individuated by type-level relations to their reciprocal partner powers, and their mutual manifestations. If $\varphi$ is, inter alia, a power to bring about an instance of $\phi$, then $\varphi$ could not be fully individuated unless $\phi$ already has at least one instance. 
ent understanding of what it is to bring it about that $p$, but logics in which (4) does not hold will result if we insist on a stricter reading that does not allow transitive cases.

\section{The Role of Powers in Grounding Modality}

On weak dispositionalism, it is not true in full generality that modality is grounded in the powers of actual things. Given the disjunctive definition of possibility $\left(\mathrm{POSS}_{3}\right.$ ), the truthmaker for $\langle\nabla p\rangle$ is either the truthmaker for $\langle p\rangle$, or the truthmaker for $\langle\exists \phi\rangle[p](\phi)\rangle$. It is possible that $2+2=4$ not in virtue of a power to bring it about that $2+2=4$, but because it is true that $2+2=4$. The truthmakers of propositions such as this one ground not only their truth, but also their possible truth. The same holds, mutatis mutandis, for $\left\langle\downarrow\left(\mathrm{E}_{\mathrm{T}}=\sum\right)>\right.$. Given the conjunctive definition of necessity $\left(\mathrm{NEC}_{3}\right)$, what makes $\langle\square(2+2=4)>$ true is whatever makes $<2+2=4>$ true and the nonexistence of a power to bring about its falsity; similarly, mutatis mutandis, for $\left\langle\square\left(E_{\mathrm{T}}=\sum\right)>\right.$. I cannot argue for it here, but I favour truthmaker non-maximalism, according to which negative existentials do not require truthmakers. If true, $\langle\exists \phi\rangle[\neg p](\phi)>$ is made true by some thing or things having the power to bring it about that $\neg p$, while its negation $\langle\neg \exists \phi\rangle[\neg p](\phi)>$, if true, is made true by the fact that there is no truthmaker for $\langle\exists \phi\rangle[\neg p](\phi)\rangle$.

It is tempting to suppose that $\langle 2+2=4>$ has a special modal status compared to $<\mathrm{E}_{\mathrm{T}}=\sum>$. After all, is the former not somehow beyond the range of powers in a way the latter is not? Suppose for the sake of argument that mathematical Platonism is true, and $<2+2=4>$ is made true by abstract mathematical objects. One might suggest that it follows from this that $\neg \exists \phi>[\neg(2+2=4)](\phi)$, while nothing of the sort follows in the case of $<\mathrm{E}_{\mathrm{T}}=\sum>$, whose truthmaker is a specific physical state of the entire universe. Given $\left(\mathrm{NEC}_{3}\right)$, however, the two propositions are necessary in just the same sense. Each is true and there is no power to bring it about that either is not, or that such a power exists. There is nothing remotely contingent about the necessity of either proposition.

Because it is the non-existence of certain powers that partially grounds the necessity of necessary propositions, it is not the case that these latter have powerful particulars as truthmakers. Despite this, weak dispositionalism is consistent with the view that powers determine what is de dicto necessary. $^{22}$ If the modal status of any true proposition were different from its actual modal status, then there would be some corresponding difference in the available powers. Had it been the case that $\mathrm{E}_{\mathrm{T}}=\sum$ but $\neg \square\left(\mathrm{E}_{\mathrm{T}}=\sum\right)$, for instance, then there would have been a power to bring it about that $\neg \mathrm{E}_{\mathrm{T}}=\sum$. On the current approach, this is a counterpossible, for weak dispositionalism 
validates $\mathrm{S} 4$. On the standard possible worlds semantics for counterfactuals, $<p \Rightarrow q>$ is true iff no possible world where $p \wedge \neg q$ is closer to actuality than the closest world where $p \wedge q$. Counterpossibles are trivially true on the standard semantics, because there are no $p$-worlds at all. There is no corresponding reason to worry about counterpossibles given dispositionalism, although it patently does not follow that a dispositionalist semantics that accommodates them can be formulated. ${ }^{23}$

The proposed amendment suggests a principled way of restricting the scope of propositional quantification in the strong dispositionalist biconditional, on which it comes out true. In weak dispositionalism, we secure formal adequacy with unrestricted propositional quantification by stipulating that truth is sufficient for possible truth. Note, however, that $\left(\mathrm{POSS}_{3}\right)$ entails that if $\langle p\rangle$ is false, then $\diamond p$ iff $\exists \phi\rangle[p](\phi)$. This suggests that the role of powers in weak dispositionalism consists in grounding the unactualized possibilities. To simplify matters, let us suppose that propositions are timeindexed, and so cannot change their truth-values. Unactualized possibilities will be represented by a proper subset of those propositions that are eternally false, for instance $<$ David Yates is a dancer on February 25 ${ }^{\text {th }}$, 2014>. Intuitively, powers determine which of the ways the world is not are ways it might have been. Where $\langle p>$ is eternally false: $\diamond p \equiv \exists \phi>[p](\phi)$. There is no prospect of a logic restricted to falsehoods, so this biconditional is not a definition of the possibility operator for a domain-specific modal logic. It does, however, specify a plausible role for powers in grounding modality, which makes it attractive that weak dispositionalism has it as a consequence.

\section{Conclusion}

In the strong form explicitly endorsed by some of its main proponents, dispositionalism is formally inadequate, but it can be fixed by means of a stipulative connection between truth and possibility. The resulting theory, weak dispositionalism, entails that if $\langle p\rangle$ is false, the power to bring it about that $p$ is both necessary and sufficient for $\langle p>$ 's possible truth, which in turn suggests that powers ground unactualized possibilities. In conclusion, I shall now suggest, the idea that truth is trivially sufficient for possibility is independently plausible. In Kripke models, in order to derive $\langle\diamond p\rangle$ from $\langle p\rangle$ and correspondingly, $\langle p\rangle$ from $\langle\square p\rangle$, we need to know that the accessibility

23 Jacobs' semantics, according to which the antecedent and consequent of true counterfactuals are related as networks of powers to their manifestations, seems unable to accommodate any counterfactuals that express synchronic non-causal determination relations. Consider, for instance, the following expression of global supervenience of mind on brain: <if there were some difference in the actual distribution of mental properties, there would be some difference in the physical state of the world $>$. 
relation $R$ is reflexive. Recall that $\langle\nabla p\rangle$ is true at $w$ iff $\langle p\rangle$ is true at some world accessible from $w$. Provided $R$ is reflexive, every instance of the schema $p \supset \diamond p$ is true, for in that case that $\langle p>$ 's truth at $w$ guarantees that $\langle p\rangle$ is true at a world accessible from $w$. This is far from trivial, in contrast with the corresponding everyday inference: once we know that $\langle p\rangle$ is true, we take its possible truth for granted. On weak dispositionalism, $<\diamond p>$ follows a fortiori from $\langle p>$ by disjunction introduction. If we suppose that the weak dispositionalist possibility operator analyses our concept of possibility, we can explain why the inference from truth to possible truth seems trivial: it is trivial. This is not an objection to Kripke semantics, which is not intended as an analysis of our modal concepts. Nonetheless, it is an attractive feature of weak dispositionalism that the definitions of the operators that it provides are in harmony with our modal reasoning practices. ${ }^{24}$

\section{Bibliography}

Cameron, R. P. (2008). 'Truthmakers and Modality', Synthese 164, pp. $261-280$.

Contessa, G. (2010). 'Modal Truthmakers and Two Varieties of Actualism', Synthese 174, pp. 341-353.

Jacobs, J. D. (2010). 'A Powers Theory of Modality-Or, How I Learned to Stop Worrying and Reject Possible Worlds', Philosophical Studies 151, pp. 227-248.

Lewis, D. (2001). 'Truthmaking and Difference-Making', Noûs 35, pp. 602-615.

Martin, C. B. (2008). The Mind in Nature. Oxford: Oxford University Press.

Pruss, A. R. (2002). 'The Actual and the Possible', in R. M. Gale (ed.) The Blackwell Guide to Metaphysics (Oxford: Blackwell), pp. 317-333. - (2011). Actuality, Possibility and Worlds. New York: Continuum.

Vetter, B. (forthcoming). 'From Potentiality to Possibility', to appear in Handbook of Potentiality, (eds.) Engelhard, K. and Quante, M., (Springer, forthcoming).

Williams, N. E. and Borghini, A. (2008). 'A Dispositional Theory of Possibility', Dialectica 62, pp. 21-41.

Williamson, T. (2005). 'Armchair Philosophy, Metaphysical Modality and Counterfactual Thinking', Proceedings of the Aristotelian Society 105, pp. 1-23. University of Nottingham, and at seminars at the LanCog Research Group, University of Lisbon, and the University of Aarhus. My thanks to the organisers, and to all those who participated for some very useful comments and criticisms. This paper is based on research funded by the European Research Council. 